\title{
Molecular-dynamics-based investigation of scattering path contributions to the EXAFS spectrum: The $\mathrm{Cr}^{3+}$ aqueous solution case
}

\author{
Patrick J. Merkling, ${ }^{1}$ Adela Muñoz-Páez, ${ }^{2}$ José M. Martínez, ${ }^{1}$ Rafael R. Pappalardo, ${ }^{1}$ and Enrique Sánchez Marcos ${ }^{1, *}$ \\ ${ }^{1}$ Departamento de Química Física, Universidad de Sevilla, 41012 Sevilla, Spain \\ ${ }^{2}$ Departamento de Química Inorgánica-ICMSE, Universidad de Sevilla-CSIC, 41012 Sevilla, Spain
}

(Received 26 March 2001; published 14 June 2001)

\begin{abstract}
Extended x-ray absorption fine structure spectra were computed based on molecular-dynamics (MD) structural data of a $\left[\mathrm{Cr}\left(\mathrm{H}_{2} \mathrm{O}\right)_{6}\right]^{3+}$ aqueous solution using nonempirical cation-water potentials. An excellent reproduction of the experimental spectrum was achieved. A simple estimation of Debye-Waller factors of the multiple-scattering paths is deduced from MD simulations. The influence of the single-scattering path due to the second hydration shell as compared with the multiple-scattering paths within the first hydration shell allows a reasonable determination of the second hydration shell distance $R\left(\mathrm{Cr}-\mathrm{O}_{\mathrm{II}}\right)$ within $0.1 \AA$.
\end{abstract}

DOI: 10.1103/PhysRevB.64.012201

PACS number(s): 61.10.Ht, 61.20.Ja

Extended x-ray absorption fine structure (EXAFS) spectroscopy provides valuable structural information to determine interatomic distances and ordering around the absorber atom. The information from this spectroscopy becomes crucial in systems where only short-range order is present and concentrations of the absorber atom are lower than $1 \%-2 \%$ in solids or $0.2 \mathrm{M}$ in solution. Under these conditions, the alternative diffraction techniques are not sensitive enough. Whereas the technique has been extensively used for problems in solid state, its application to aqueous solutions has been infrequent. ${ }^{1}$ This is partly justified by the fact that it has been generally thought that no more than one hydration shell around the absorber atom could be determined in the liquid. ${ }^{2}$

Our group proposed that it is possible to determine the second hydration shell for the case of highly charged transition-metal cations forming stable solvates such as $\mathrm{Cr}^{3+}$ salts in water. ${ }^{3,4}$ This makes the attractive determination of cationic environment beyond the first coordination shell possible, not only in pure aqueous solutions but also in biochemical media where metal cations play a significant role. However, scattering phenomena involving more than one atom (see Fig. 1) may interfere with single scattering (SS) phenomena due to the second shell, since $R\left(\mathrm{M}-\mathrm{O}_{\mathrm{II}}\right)$ $\approx 2 R\left(\mathrm{M}-\mathrm{O}_{\mathrm{I}}\right){ }^{6,5}$ This situation is not new in EXAFS studies of solutions, but it makes the determination dependent on a large number of fit parameters of a different physical nature (structural, spectroscopic, and dynamic) which compelled several authors to include additional and EXAFSindependent information by using computer simulations. ${ }^{7-13}$ In these studies, the Fourier transform (FT) of the EXAFS spectrum is often compared with radial distribution functions or, alternatively, direct simulations of EXAFS spectra are performed on the basis of structural results and compared with the experimental spectra. Conversely, this type of comparison has also been suggested as a way of checking the reliability of the intermolecular potentials. ${ }^{9,12}$ Our group proposed the use of the hydrated ion concept to build nonempirical intermolecular potentials of highly charged metal cations of the type $\left[\mathrm{M}\left(\mathrm{H}_{2} \mathrm{O}\right)_{n}\right]^{m+}-\mathrm{H}_{2} \mathrm{O}$. One of the key points of this study is the use of first-principles $a b$ initio intermolecular potentials to deal with the important many-body terms present in the strong ion-solvent interactions. ${ }^{14}$ These potentials are able to describe fairly well structural, dynamical, energetic, and spectroscopic properties of ionic solutions. ${ }^{15-17}$ The accuracy in the prediction of structural parameters involved in the first and second hydration shells as well as in the vibrational frequencies associated to the first hydration shell is particularly relevant for the present paper. ${ }^{16}$

This paper presents a theoretical modeling of the EXAFS spectrum of an aqueous chromium salt solution in order to get a deeper insight into the determination of the second hydration shell in one of the most representative stable hydrated cations. ${ }^{1}$ This is done on the basis of moleculardynamics (MD) simulations that use the specially designed $\left[\mathrm{Cr}\left(\mathrm{H}_{2} \mathrm{O}\right)_{6}\right]^{3+}-\mathrm{H}_{2} \mathrm{O}$ potential ${ }^{16}$ and the current version of the FEFF program. ${ }^{18}$ Results are compared with an experimental EXAFS spectrum of an aqueous $\mathrm{Cr}\left(\mathrm{NO}_{3}\right)_{3}$ solution recently published. ${ }^{5}$

A simplified expression of the EXAFS function is given by

$\chi(k)=\sum_{j}^{\text {paths }} \frac{N_{j} S_{0}^{2}}{k R_{j}^{2}}|f(k)| \sin \left[2 k R_{j}+\varphi_{j}(k)\right] \times e^{-2 R_{j} / \lambda} e^{-2 \sigma_{j}^{2} k^{2}}$

Scattering paths:

Single

FIG. 1. Main scattering paths contributing to the EXAFS spectrum: First and second hydration shell SS and first shell MS at the oxygen sites. 
where the summation goes over all paths $j$ involving either single (SS) or multiple-scattering (MS) phenomena. In this equation, $k$ is the wave vector, $N_{j}$ the coordination number, $S_{0}^{2}$ the amplitude reduction factor, $R_{j}$ the path length, $f$ the curved-wave backscattering amplitude, $\varphi_{j}$ the phase shift, $\lambda$ the mean-free-path, and $\sigma_{j}^{2}$ the Debye-Waller (DW) factor. The disorder is thus expressed through DW factors, which lead to an intensity loss of the EXAFS signal. In an alternative equation, the disorder arises from summing over a number of MD structural arrangements. This procedure enables the sampling of the configurational space:

$$
\begin{aligned}
\chi(k)= & \frac{1}{N_{s}} \sum_{i}^{\text {struc. paths }} \sum_{j^{\prime}} \frac{N_{j^{\prime}} S_{0}^{2}}{k R_{i j^{\prime}}^{2}}|f(k)| \\
& \times \sin \left[2 k R_{i j^{\prime}}+\varphi_{j^{\prime}}(k)\right] e^{-2 R_{i j^{\prime}} / \lambda},
\end{aligned}
$$

where $N_{s}$ is the number of structures considered, $i$ goes over the structures obtained from the statistical sampling, and $j^{\prime}$ over the paths.

Structural parameters were obtained from an experimental spectrum using Eq. (1) and the same procedure was applied to the theoretical spectrum computed by means of Eq. (2). A previous study by Campbell et al. on the same system focuses on the way of obtaining DW factors from MD simulations and determines a theoretical EXAFS spectrum by applying an equation of type $1 .^{10}$

Cr $K$-edge EXAFS spectra were calculated from snapshots of MD simulations of $\mathrm{Cr}^{3+}$ in water using a new version (2.16) of the MOLDY program. ${ }^{19}$ A flexible hydrated ion model was employed such that $\mathrm{Cr}^{3+}-\left(\mathrm{H}_{2} \mathrm{O}\right)_{\mathrm{I}}$ interactions were described by an effective IW1 potential including many-body interactions of the whole first hydration shell, ${ }^{16}$ whereas $\left[\mathrm{Cr}\left(\mathrm{H}_{2} \mathrm{O}\right)_{6}\right]^{3+}-\left(\mathrm{H}_{2} \mathrm{O}\right)_{\text {bulk }}$ interactions were described by the HIW potential. ${ }^{20}$ Bulk water-water interactions were described by the TIP4P potential. The system was formed by $1 \mathrm{Cr}^{3+}+6\left(\mathrm{H}_{2} \mathrm{O}\right)_{\mathrm{I}}+512\left(\mathrm{H}_{2} \mathrm{O}\right)_{\text {bulk }}$. 1 ns of simulation at $298 \mathrm{~K}$ was performed in the microcanonical ensemble. ${ }^{23}$ The sampling interval for successive snapshots was set to 2 ps. For each structure, a cutoff centered around the chromium atom was applied such that two hydration shells were explicitly considered to calculate the EXAFS function from selected snapshots by means of the FEFF program (version 8.10). ${ }^{18}$ The subsequent application of FEFFIT $^{21}$ (version 2.54) yielded the spectra in $k$ and $r$ spaces. The spectra presented in Fig. 2 correspond to the average of 500 individual spectrum computations, according to Eq. (2). Whereas hydrogen atoms were taken into account to compute the potentials of oxygen atoms, no scattering paths including hydrogen atoms were considered, because of their low scattering intensities.

The theoretical EXAFS averaged spectrum is compared with the experimental spectrum recently published for a 0.1 $\mathrm{M} \mathrm{Cr}\left(\mathrm{NO}_{3}\right)_{3}$ solution. ${ }^{5}$ The modulus of both Fourier transforms $(|\mathrm{FT}|)$ are plotted in Fig. 2(a). The only parameter empirically added to the theoretical spectrum is $S_{0}^{2}$, its value being set to 0.81 in order to reproduce the intensity of the first peak. It is seen that theoretical and experimental spectra show an excellent agreement, not only for the principal peak,
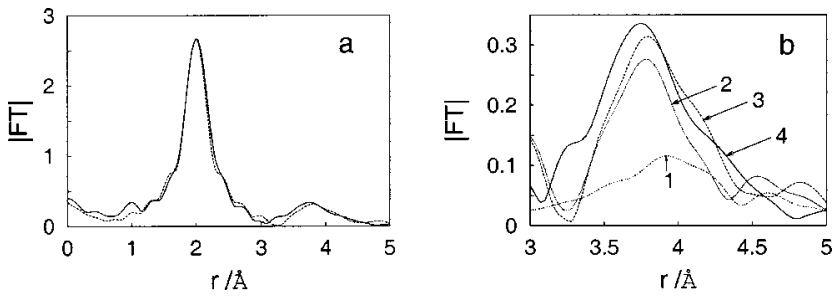

FIG. 2. (a) $\mathrm{Cr}-\mathrm{O}$ phase corrected FT of the EXAFS spectrum from experiment (full) and from MD simulation (dashed). (b) Theoretical contributions to the second peak of the FT; second shell SS (1), second shell SS + first shell MS (2), all paths (3). Experimental (4) curve shown for comparison.

but also for the second peak. To investigate the main contributions to this second peak, Fig. 2(b) shows the $|\mathrm{FT}|$ in the $3-5 \AA$ region. In addition to the global curves as in Fig. 2(a), two theoretical curves computed from the inclusion of selected paths are shown: the first one (1) is generated when only second hydration shell SS paths are considered, and the second curve (2) is obtained by the addition of first shell MS paths. The number of individual paths contributing to the second shell SS is roughly ten times smaller than the number of first shell MS contributions. Second hydration shell SS paths represent roughly $35 \%$ of this second peak, whereas the addition of the paths defined within the first hydration shell makes up roughly $85 \%$ of the intensity of this peak. Campbell et al. obtain a similar estimation of the second shell contribution from their study. ${ }^{10}$

The use of Eq. (1) implies the definition of a reference (averaged) structure described by $N_{j}$ and $R_{j}$ and a statistical distribution of geometrical fluctuations accounted for by the DW factor. Thus, there is a unique structure for which several SS and MS paths can be defined. The use of Eq. (2) implies the consideration of a high number of different structures and for each one several SS and MS paths are defined. The scope of this study is to discuss the results obtained using Eq. (2) within the usual EXAFS frame of Eq. (1). The large number of slightly different paths involved in the scattering phenomenon had to be rationalized. To this aim, the MD structural information was classified in a reduced number of paths, and the geometrical criteria for pertaining to a given path were (i) angular deviations from the averaged geometry smaller than $15^{\circ}$ and (ii) for first shell paths, $R(\mathrm{Cr}-\mathrm{O}) \leqslant 3.0 \AA$. The most significant paths are shown in Fig. 1. With respect to the symmetric distribution around the $\mathrm{Cr}^{3+}$, only a $0.5 \%$ of the corresponding structural arrangements could not be ascribed to some of the previous paths. Second shell oxygen atoms were defined to lie between 3.0 and $4.5 \AA$. The mean second shell coordination number obtained is 13.9 .

Since DW factors are the mean-square displacements of path lengths $\left[\sigma_{i}^{2}=\left\langle\left(R_{i}-\langle R\rangle\right)^{2}\right\rangle\right]$, they can be obtained directly from the simulation. The results derived from the MD simulations for the most relevant paths are included in Table I. The values of DW factors for first (ss1) and second (ss2) shell SS paths are 10\%-20\% lower than those obtained by Campbell et al. ${ }^{10}$ This small difference is probably attributable to the use of a different intermolecular potential. Table I 
TABLE I. Distances and Debye-Waller factors obtained from MD simulation, and from fitting either to the computed EXAFS spectrum or to the experimental one.

\begin{tabular}{lcccccc}
\hline \hline & \multicolumn{2}{c}{ MD } & \multicolumn{5}{c}{ EXAFS fitting } \\
& & & \multicolumn{2}{c}{ Computed } & \multicolumn{2}{c}{ Experiment } \\
\cline { 2 - 7 } Path & $\mathrm{R} / \AA$ & $\sigma^{2} / \AA^{2}$ & $\mathrm{R} / \AA$ & $\sigma^{2} / \AA^{2}$ & $\mathrm{R} / \AA$ & $\sigma^{2} / \AA^{2}$ \\
\hline ss1 & 2.00 & 0.0024 & 2.00 & 0.0024 & 1.97 & 0.0025 \\
& \pm 0.003 & \pm 0.00006 & \pm 0.001 & \pm 0.0001 & \pm 0.001 & \pm 0.0003 \\
ss2 & 4.08 & 0.039 & 3.96 & 0.045 & 3.95 & 0.027 \\
& \pm 0.01 & \pm 0.0006 & \pm 0.02 & \pm 0.004 & \pm 0.03 & \pm 0.006 \\
A & 4.00 & 0.0043 & 4.00 & 0.0048 & 3.94 & 0.0050 \\
B & 4.00 & 0.0043 & 4.00 & 0.0048 & 3.94 & 0.0050 \\
C & 4.00 & 0.0096 & 4.00 & 0.0096 & 3.94 & 0.0100 \\
D & 3.41 & 0.0045 & 3.41 & 0.0048 & 3.37 & 0.0050 \\
\hline \hline
\end{tabular}

${ }^{\mathrm{a}}$ EXAFS data from Ref. 5.

shows that four paths, A, B, C, and ss2, have very similar distances. This is the main reason for the difficulty in obtaining the structural information from the second coordination sphere, as already pointed out in the Introduction. Interestingly, the DW factors calculated from the MD simulations obey quite simple relationships: $\sigma_{\mathrm{A}}^{2} \approx \sigma_{\mathrm{B}}^{2} \approx \sigma_{\mathrm{D}}^{2} \approx 2 \sigma_{\mathrm{ss} 1}^{2}$. In the case of the collinear paths $\mathrm{A}$ and $\mathrm{B}$, this agrees with the independent vibration model proposed by Yokoyama et al. ${ }^{22}$ According to the definition of $\sigma^{2}, \sigma_{\mathrm{C}}^{2}=4 \sigma_{\mathrm{ss} 1}^{2}$. The triangular path D shows a DW factor close to that of the collinear paths, which is consistent with an extension of the independent vibration model. Since this path is much shorter than paths $\mathrm{A}, \mathrm{B}, \mathrm{C}$, and ss2, the maximum of the second peak in the FT [curves (2) and (3) in Fig. 2(b)] is shifted about $-0.1 \AA$ with respect to the peak due to the ss 2 path [curve (1)]. The set of DW factors associated to the first shell presents small values as a consequence of the fact that the first shell oxygen atoms are confined in a narrow region around the cation.

EXAFS data analyses were performed to examine the strategy used to determine the second hydration shell. For this purpose, two analyses were carried out. In the first one, the computed spectrum previously generated through Eq. (2) was employed as input data for a fitting procedure. The parameters obtained are displayed in columns 4 and 5 of Table I. In the second analysis, the experimental spectrum was fitted, and the results appear in columns 6 and 7. In both analyses, the spectrum was decomposed in scattering paths contributions $^{5,22}$ from the assumption of a model geometry corresponding to a symmetric $\left[\mathrm{Cr}\left(\mathrm{H}_{2} \mathrm{O}\right)_{6}\right]^{3+} \cdot\left(\mathrm{H}_{2} \mathrm{O}\right)_{12}$ cluster. The first and second shell SS paths, as well as all the first shell MS paths, were included in the fitting procedure. To reduce the number of parameters to be optimized, DW factors for the first shell MS paths were correlated with the DW factor of the first shell SS path according to the ratio found in the MD simulation. The consideration of third-order cumulants did not improve the fit. The $S_{0}^{2}$ value was fixed to 0.81 . Therefore, the free parameters were the $R\left(\mathrm{Cr}-\mathrm{O}_{\mathrm{I}}\right)$ and $R\left(\mathrm{Cr}-\mathrm{O}_{\mathrm{II}}\right)$ distances, their corresponding $\mathrm{DW}$ factors, the inner potential correction $\Delta E_{0}$, and the experimental broaden- ing $E_{i} \cdot{ }^{24}$ The fit yields good $\mathcal{R}$ factors in both cases ( $\mathcal{R}=0.013$ for the experimental, $\mathcal{R}=0.001$ for the computed).

A first analysis of Table I concerns the comparison of the results derived from the computed spectrum and those coming from the structural analysis of the MD simulations. At first sight, they are expected to match. This is the case for $R\left(\mathrm{Cr}-\mathrm{O}_{\mathrm{I}}\right)$ and $\sigma_{\mathrm{ss} 1}^{2}$ values. However, the fitting procedure included a certain number of approximations that led to predict a $R\left(\mathrm{Cr}-\mathrm{O}_{\mathrm{II}}\right)$ value $0.12 \AA$ shorter than the averaged value obtained directly from the MD structures. Likewise, the fitted $\sigma_{\mathrm{ss} 2}^{2}$ value is slightly larger than the MD one. These discrepancies in the second shell description can be envisaged as a methodological error associated to the shortcomings of the fitting procedure of a nonprincipal contribution to the EXAFS signal. This causes an uncertainty in the determination of the second shell distance of $\pm 0.1 \AA$.

A second point consists in the twofold comparison of the results obtained by fitting the experimental spectrum with those obtained either by fitting the computed one or the structural $\mathrm{MD}$ results. The $R\left(\mathrm{Cr}-\mathrm{O}_{\mathrm{I}}\right)$ value only differs by $0.03 \AA$ from the fitted experimental data to both the computed spectrum and the MD simulation. This difference could be ascribed to the intermolecular potentials employed. The DW factor for the first shell is the same in all cases within the uncertainty on its determination. The $R\left(\mathrm{Cr}-\mathrm{O}_{\mathrm{II}}\right)$ value is $0.13 \AA$ shorter than the MD result, but only $0.01 \AA$ shorter than the value derived from the fitting of the computed spectra. Bearing in mind the small differences observed for the first shell, this discrepancy in the $R\left(\mathrm{Cr}-\mathrm{O}_{\mathrm{II}}\right)$ value may be due to the whole fitting procedure. Two main causes could be invoked to understand it: first, the inability of the model geometry used as reference to bend, and second, the restriction in the path number taken into account in the fitting.

In conclusion, the EXAFS spectra generated from a MDbased simulation, that uses first-principles hydrated ionwater potentials and the FEFF code, reproduces very well the experimental spectrum considering the hydration structure up to the second shell. This confirms simultaneously the good behavior of the $\mathrm{Cr}^{3+} \mathrm{HIW}$ intermolecular potential and of the FEFF code. EXAFS-independent analysis of the structural information derived from the MD computations supports the application of the independent vibration model to estimate DW factors of MS paths. An intrinsic uncertainty in the methodology to determine the second hydration shell of $\pm 0.1 \AA$ has been detected. The value of $\mathrm{S}_{0}^{2}$ in aqueous chromium solutions has been determined to be 0.81 . It appears that basic and easily applicable rules for the extraction of structural parameters from experimental EXAFS spectra of new systems in solution may be formulated from the outcome of computer simulations.

This work was supported by the Fundación Ramón Areces (XI Concurso Nacional). We thank Professor J. J. Rehr and Dr. K. Refson for supplying us with the latest versions of the FEFF and MOLDY programs, respectively. 
*Email address: sanchez@simulux.us.es

${ }^{1}$ H. Ohtaki and T. Radnai, Chem. Rev. 93, 1157 (1993).

${ }^{2}$ M. Magini, G. Licheri, G. Paschina, G. Piccaluga, and G. Pinna, $X$-Ray Diffraction of Ions in Aqueous Solutions: Hydration and Complex Formation (Chemical Rubber, Boca Raton, FL, 1988).

${ }^{3}$ A. Muñoz-Páez and E. Sánchez Marcos, J. Am. Chem. Soc. 114, 6931 (1992).

${ }^{4}$ A. Muñoz-Páez, R.R. Pappalardo, and E. Sánchez Marcos, J. Am. Chem. Soc. 117, 11710 (1995).

${ }^{5}$ H. Sakane, A. Muñoz-Páez, S. Díaz-Moreno, J.M. Martínez, R.R. Pappalardo, and E. Sánchez Marcos, J. Am. Chem. Soc. 120, 10397 (1998).

${ }^{6}$ A. Filiponi, P. D’Angelo, N.V. Pavel, and A. Di Cicco, Chem. Phys. Lett. 225, 150 (1994).

${ }^{7}$ S.J. Gurman and R.L. McGreevy, J. Phys.: Condens. Matter 2, 9463 (1990).

${ }^{8}$ B.J. Palmer, D.M. Pfund, and J.L. Fulton, J. Phys. Chem. 100, 13393 (1996).

${ }^{9}$ D. Roccatano, H.J.C. Berendsen, and P. D'Angelo, J. Chem. Phys. 108, 9487 (1998), and references therein.

${ }^{10}$ L. Campbell, J.J. Rehr, G.K. Schenter, M.I. McCarthy, and D. Dixon, J. Synchrotron Radiat. 6, 310 (1999).

${ }^{11}$ M. Ablanov, H. Matsuura, and R. Takagi, Electrochemistry 67, 839 (1999).

${ }^{12}$ M.M. Hoffmann, J.G. Darab, B.J. Palmer, and J.L. Fulton, J. Phys. Chem. A 103, 8471 (1999).

${ }^{13}$ D. Spångberg, K. Hermansson, P. Lindqvist-Reis, F. Jalilehvand, M. Sandström, and I. Persson, J. Phys. Chem. B 104, 10467 (2000).
${ }^{14}$ M.J. Elrod and R.J. Saykally, Chem. Rev. 94, 1975 (1994).

${ }^{15}$ R.R. Pappalardo, J.M. Martínez, and E. Sánchez Marcos, J. Phys. Chem. 100, 11748 (1996).

${ }^{16}$ J.M. Martínez, R.R. Pappalardo, and E. Sánchez Marcos, J. Chem. Phys. 109, 1445 (1998).

${ }^{17}$ J.M. Martínez, R.R. Pappalardo, and E. Sánchez Marcos, J. Am. Chem. Soc. 121, 3175 (1999).

${ }^{18}$ A. Ankudinov, B. Ravel, J.J. Rehr, and S.D. Conradson, Phys. Rev. B 58, 7565 (1998).

${ }^{19}$ K. Refson, Comput. Phys. Commun. 126, 310 (2000).

${ }^{20}$ J.M. Martínez, R.R. Pappalardo, E. Sánchez Marcos, K. Refson, S. Díaz-Moreno, and A. Muñoz-Páez, J. Phys. Chem. B 102, 3272 (1998).

${ }^{21}$ E.A. Stern, M. Newville, B. Ravel, Y. Yacoby, and D. Haskel, Physica B 208\&209, 117 (1995).

${ }^{22}$ T. Yokoyama, T. Ohta, and H. Sato, Phys. Rev. B 55, 11320 (1997), and references therein.

${ }^{23}$ Periodic boundary conditions were applied. The Ewald sum was applied for Coulombic interactions. A spherical cutoff of $15 \AA$ for short range potentials and for the real part of the Ewald sum were applied. The symplectic integration algorithm of the leapfrog type implemented in the MOLDY program enabled us to increase the time step to $2.0 \mathrm{fs}$.

${ }^{24}$ Paths of up to 6 legs and with a length of up to $5 \AA$ were included. Thresholds for full curved and plane wave filters are $2.0 \%$ and $1.0 \%$, respectively. Fitting conditions: $k^{2}, \Delta R_{\text {Fit }}$ $=0.9-4.5 \AA$, and $\Delta k_{F T}=2.8-13.7 \AA^{-1} .\left(\Delta E_{0}=0.4 \mathrm{eV}, E_{i}\right.$ $=-0.45 \mathrm{eV})$ for "computed"; $\left(\Delta E_{0}=6.0 \mathrm{eV}, E_{i}=0.6 \mathrm{eV}\right)$ for "experimental.", 